\title{
PD-1: Dual guard for immunopathology
}

\author{
Hye Ryun Kim, Hyo Jin Park, and Sang-Jun Ha
}

Despite remarkable medical development, chronic infectious diseases caused by the infections with persistent pathogens such as hepatitis B virus (HBV), hepatitis C virus (HCV), human immunodeficiency virus (HIV), and mycobacterium tuberculosis (M.tb), have not been conquered yet. During the chronic infection, the interaction between host and chronic pathogen often fosters immune suppressive environment. Such environment leads to the exhaustion of pathogen-specific T cells, resulting in the failure to the eradication of pathogen but the success to the protection of host from immunopathological damage. Finally, host and pathogen ensure the reduction of host immunopathology but the extension of pathogen persistence.

Under the persistence of pathogens, effector $\mathrm{T}\left(\mathrm{T}_{\mathrm{eff}}\right)$ cells lose progressively their effector function such as cytokine production and proliferative potential and finally become exhausted $\mathrm{T}\left(\mathrm{T}_{\text {exh }}\right)$ cells. T-cell exhaustion can be caused by various factors including immune checkpoints, cytokines, regulatory $\mathrm{T}\left(\mathrm{T}_{\mathrm{reg}}\right)$ cells, and altered antigen presenting cells (APCs) [1]. Immune inhibitory receptors, also known as immune checkpoints, such as programmed death-1 (PD-1), T-cell immunoglobulin mucin 3 (TIM3 ), cytotoxic $\mathrm{T}$ lymphocyte antigen-4 (CTLA-4), and lymphocyte activation gene-3 (LAG-3) are expressed on $\mathrm{T}_{\text {eff }}$ cells by $\mathrm{T}$-cell receptor (TCR) stimulation and their expressions are maintained or even increased by repeated TCR stimulation during chronic infection. Upon ligands binding, such checkpoints are triggered to transmit inhibitory signal into $\mathrm{T}_{\text {eff }}$ cells by alone or their combination, which leads to the generation of $\mathrm{T}_{\mathrm{exh}}$ cells and the attenuation of T-cell-mediated immunopathology. The interaction of PD-1 and its ligand, PD-L1, has been known to play a critical role in the exhaustion of $\mathrm{T}_{\text {eff }}$ cells. The role of PD-1 during chronic pathogen infection in suppressing T-cell function was demonstrated in the mouse model chronically infected with lymphocytic choriomeningitis virus (LCMV) [2]. This study showed that blockade of PD-1:PD-L1 interaction during chronic LCMV infection controlled viremia by restoring the function of virusspecific $\mathrm{T}_{\text {exh }}$ cells. More specifically, only PD- $1^{+} \mathrm{CD} 8^{+} \mathrm{T}$ cells, but not PD-1- $\mathrm{CD} 8^{+} \mathrm{T}$ cells, regained their function upon PD-1:PD-L1 blockade, indicating that the effect of PD-1 on T-cell exhaustion is T-cell-intrinsic (Figure 1). Another interesting point was that PD-L1-deficient mice succumbed to chronic LCMV infection, resulting in death of mice within one week post-infection. PD-1:PD-L1 pathway seemed to be also critical in protecting mice from persistent bacterial infection because PD-1-deficient mice were extraordinarily sensitive to tuberculosis [3]. These data strongly suggests that PD-1:PD-L1 pathway regulates immune-mediated tissue damage during persistent infection by paralyzing the pathogen-specific $\mathrm{T}$ cells.

The claim that PD-1 expressed by $\mathrm{T}$ cells is a culprit of T-cell exhaustion is now generally accepted but there can be another causing factors. One of the candidates is $\mathrm{T}_{\text {reg }}$ cells, which play an important role for maintaining immunological self-tolerance and controlling T-cell-mediated autoreactive T-cell attack on tissue, because a number of studies showed the increase of $\mathrm{T}_{\text {reg }}$ cell population during persistent viral, helminthic, and bacterial infections. Depletion of $\mathrm{T}_{\text {reg }}$ cells in the mice chronically infected with LCMV was reported to strikingly expand functional LCMV-specific CD8 ${ }^{+} \mathrm{T}$ cells,

\section{Chronic pathogen infection}
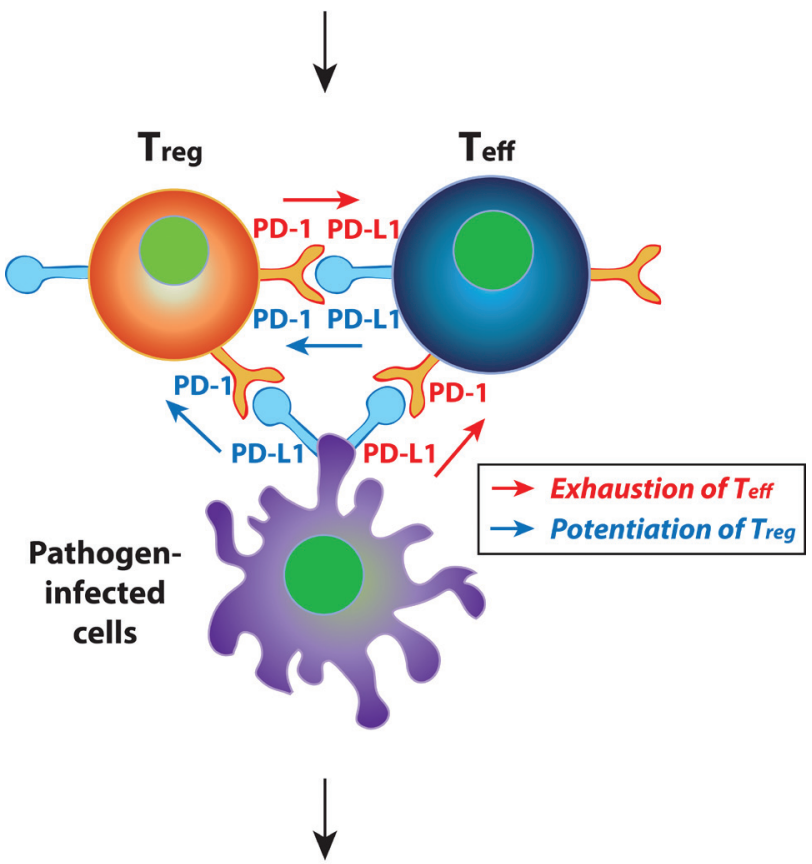

\section{Suppression of T cell response Attenuation of immunopathology}

Figure 1: PD-1:PD-L1 pathway as a dual guard for the protection of immunopathological damage. $\mathrm{PD}-1$ is highly upregulated on $\mathrm{T}_{\text {reg }}$ cells as well as $\mathrm{T}_{\text {eff }}$ cells during chronic pathogen infection. PD-1:PD-L1 interactions between $\mathrm{T}_{\text {eff }}$ cells and pathogen-infected cells or between $\mathrm{T}_{\text {reg }}$ cells and $\mathrm{T}_{\text {eff }}$ cells provide $\mathrm{T}_{\text {eff }}$ cell exhaustion signal or $\mathrm{T}_{\text {reg }}$ cell potentiation signal, thereby attenuating immunopathology. 
suggesting the role of $\mathrm{T}_{\text {reg }}$ cells in the suppression of T-cell immune response during persistent pathogen infection [4]. However, this temporal ablation of $\mathrm{T}_{\text {reg }}$ cells failed to not only diminish viremia but promote immunopathology because it also triggered upregulation of PD-L1 on LCMV-infected cells, which again delivers negative signal to PD-1-expressing $\mathrm{T}$ cells. It has been widely known that $\mathrm{T}_{\text {reg }}$ cells constitutively express some immune checkpoints such as CTLA-4 and LAG-3. Of particular interest, like $\mathrm{T}_{\text {exh }}$ cells, $\mathrm{T}_{\text {reg }}$ cells during chronic pathogen infection was reported to further upregulated immune checkpoints including CTLA-4, LAG-3, PD-1, and TIM$3[4,5,6]$. However, while $\mathrm{T}_{\text {eff }}$ or $\mathrm{T}_{\text {exh }}$ cells-expressed immune checkpoints debilitate their effector function, $\mathrm{T}_{\text {reg }}$ cells-expressed ones, especially CTLA-4, have been reported to potentiate their suppressor function in direct or indirect way. For instance, CTLA-4 expressed by $\mathrm{T}_{\text {reg }}$ cells modulates dendritic cells (DCs), thereby enhancing $\mathrm{T}_{\text {reg }}$ cell suppressive function as positive regulator [7]. Taken together, albeit such a complexity, it is evident that $\mathrm{T}_{\text {res }}$ cells can contribute to the inhibition of T-cell response and the protection of tissue from T-cell-mediated pathology.

In spite of the observation that PD-1 is upregulated on $\mathrm{T}_{\text {reg }}$ cells during chronic infection, it is less known about the role of PD-1 expressed by $\mathrm{T}_{\text {reg }}$ cells. We recently found that PD- $1^{\text {hi }} \mathrm{T}_{\text {reg }}$ cells generated during chronic LCMV infection displayed much stronger suppressive activity than PD- $1^{\text {lo }} \mathrm{T}_{\text {reg }}$ cells present in steady state [6]. Either PD-1 blockade on PD- $1^{\text {hi }} \mathrm{T}_{\text {reg }}$ cells or PD-L1 deficiency on $\mathrm{T}_{\text {eff }}$ cells dramatically ablated $\mathrm{T}_{\text {reg }}$ cell-mediated suppression of $\mathrm{T}_{\text {eff }}$ cell immune response, demonstrating the necessity of PD-1 on $\mathrm{T}_{\text {reg }}$ cells and PD-L1 on $\mathrm{T}_{\text {eff }}$ cells. These results highlight a critical role of PD-1:PD-L1 interaction between $\mathrm{T}_{\text {reg }}$ cells and PD-L1 on $\mathrm{T}_{\text {eff }}$ cells and define PD-1 upregulated on $\mathrm{T}_{\text {reg }}$ cells as a prerequisite for $\mathrm{T}_{\text {reg }}$ cell-mediated strong suppression (Figure 1). It should be further investigated for the molecular mechanism by which whether a ligation of PD-L1 in $\mathrm{T}_{\text {eff }}$ cells onto PD$1^{\text {hi }} \mathrm{T}_{\text {reg }}$ cells triggers PD-1 signal in $\mathrm{T}_{\text {reg }}$ cells or a reverse signal via PD-L1 in $\mathrm{T}_{\text {eff }}$ cells upon a ligation of PD-1 provided by $\mathrm{T}_{\text {reg }}$ cells inhibits $\mathrm{T}_{\text {eff }}$ cell function. If the former is applied in vivo, PD-L1 is probably provided by pathogen-infected APCs as well as $\mathrm{T}_{\text {eff }}$ cells. Regarding cell-specific expression of PD-1, our results propose that
PD-1 expressed by $T_{\text {reg }}$ cells, in addition to that by $T_{\text {exh }}$ cells, contributes to the functional suppression of $\mathrm{T}_{\text {eff }}$ cells and subsequent attenuation of immunopathology (Figure 1).

Collectively, PD-1:PD-L1 interaction is a major strategy for the suppression of $\mathrm{T}_{\text {eff }}$ cell-mediated immune response during chronic pathogen infection. Meanwhile, in regard to the immunopathological damage, PD-1:PD-L1 interaction protects $T_{\text {eff }}$ cell-mediated tissue damage in the host. Of importance, PD-1:PD-L1 interaction is capable to occur in between $T_{\text {reg }}$ cells and $T_{\text {eff }}$ cells, in addition to between $\mathrm{T}_{\text {eff }}$ cells and pathogen-infected cells. In both case, $\mathrm{T}_{\text {eff }}$ cells become exhausted via direct interaction with separate partners and eventually fail to not only eradicate pathogens but also induce immunopathology in the host. Lastly, these studies provide perspectives regarding the practical and clinical treatment strategies for chronic infectious disease. Blockade of PD-1:PD-L1 can be useful to treat efficiently chronic virus infection but its potential risk for immunopathologic damage needs to be carefully monitored.

Sang-Jun Ha: Department of Biochemistry, College of Life Science \& Biotechnology, Yonsei University, Seoul, Korea.

Correspondence to: Sang-Jun Ha, email sjha@yonsei.ac.kr

Keywords: Immunology and Microbiology Section, Immune response, Immunity

Received: July 02, 2015

Published: July 05, 2015

\section{REFERENCES}

1. Virgin HW, et al. Cell. 2009; 138:30-50.

2. Barber DL, et al. Nature. 2006; 439:682-687.

3. Lazar-Molnar E, et al. PNAS. 2010; 107:13402-13407.

4. Penaloza-MacMaster P, et al. J Exp Med. 2014; 211:19051918.

5. Punkosdy GA, et al. PNAS. 2011; 108:3677-3682.

6. Park HJ, et al. J Immunol. 2015; 194:5801-5811.

7. Wing K, et al. Science. 2008; 322:271-275. 